\title{
Interleukin-17 inhibits the growth of oral squamous cell carcinoma by promoting the differentiation of $T$ helper 17 cells
}

\author{
Yipeng Ren ${ }^{1 \#}$, Juntao Ma ${ }^{1,2 \#}$, Tong Wang ${ }^{3}$, Rongfa Bu ${ }^{1}$, Xiangpan Kong ${ }^{4}$, Yueyi Shi ${ }^{1}$, Lei Zhang ${ }^{1}$ \\ ${ }^{1}$ Department of Stomatology, Chinese PLA General Hospital, Beijing 100853, China; ${ }^{2}$ Department of Stomatology, The First Affiliated Hospital \\ of Dalian Medical University, Dalian 116011, China; ${ }^{3}$ School of Medicine, Nankai University, Tianjin 300071, China; ${ }^{4}$ Department of Oral and \\ Maxillofacial-Head and Neck Surgery, Beijing Stomatological Hospital, Capital Medical University, Beijing 100050, China \\ Contributions: (I) Conception and design: R Bu, L Zhang; (II) Administrative support: Y Shi, L Zhang; (III) Provision of study materials or patients: \\ Y Ren, J Ma, T Wang; (IV) Collection and assembly of data: Y Ren, J Ma, X Kong; (V) Data analysis and interpretation: Y Ren, J Ma, L Zhang; (VI) \\ Manuscript writing: All authors; (VII) Final approval of manuscript: All authors. \\ \#These authors contributed equally to this work. \\ Correspondence to: Lei Zhang. Department of Stomatology, Chinese PLA General Hospital, Beijing 100853, China. Email:dymajuntao2016@126.com.
}

Background: The infiltration of T helper 17 (Th17) cells has been reported in multiple solid tumors. Recent studies implicated that Th17 cells played an inhibitory role in the tumor growth. However, if Th17 cells infiltrated and played a role in oral squamous cell carcinoma (OSCC) remained unknown.

Methods: Fluorescence activated cell sorting (FACS) was performed to confirm the increase of Th17 cells in the peripheral blood and the infiltration in tumor samples from OSCC patients. To mimicking the effect of Th17 cells on OSCC growth, an OSCC line stably expressing human interleukin-17A (IL-17A) was established. Human-PBL-SCID-beige mice were employed as the hosts for the implantation of OSCC/IL17A cells. Quantitative PCR, ELISA and Western blotting were performed to analyze Th17 differentiation and function in the implanted tumors.

Results: The percentage of Th17 cells increased significantly in both the peripheral blood and the tumor tissues from OSCC patients. Expression of IL-17A promoted the differentiation of Th17 cells and suppressed the growth of implanted OSCC tumors. The transcription as well as the protein levels of IL-6 and granzyme B in the implanted OSCC cells was remarkably elevated by IL-17A expression.

Conclusions: The increased infiltration of Th17 cells was suggested to result in a therapeutic effect on OSCC as Th17 cells did in other tumors. IL-17 could promote the differentiation of Th17 cells by increasing IL-6 expression. The inhibitory effect of Th17 cells on OSCC growth was associated with their increased cytotoxic activity.

Keywords: T helper 17 (Th17); oral squamous cell carcinoma (OSCC); interleukin-17; tumor implantation; cytokines

Submitted Feb 11, 2018. Accepted for publication May 25, 2018.

doi: $10.21037 /$ tcr.2018.06.05

View this article at: http://dx.doi.org/10.21037/tcr.2018.06.05

\section{Introduction}

The tumors in head and neck are usually characterized by the progressive malignancy and occupy $6 \%$ of the newly diagnosed neoplasms (1). Approximately, 95\% cases of the head and neck tumors are squamous cell carcinoma $(2,3)$. Even taking the advancements in surgery, radiology and chemotherapy into account, the prognosis of the squamous cell carcinoma is still pessimistic because of the terribly high ratio of relapse (4). The infiltration of lymphocytes in head and neck tumors has been reported in many studies $(5,6)$, however, the exact roles of the tumor infiltrated lymphocytes (TIL) were still unclear. Moreover, since previous studies mainly focused on nasopharyngeal cancers, 
if the infiltration of TIL exists in oral squamous cell carcinoma (OSCC) is also in debated.

T helper 17 (Th17) cell is a $\mathrm{IL}-17^{+} \mathrm{CD}^{+}$subpopulation of $\mathrm{T}$ helper cells discovered recently (7). Th17 cell plays critical roles in mucosal immunity and autoimmunity, such as multiple sclerosis, immune enteritis and psoriasis (7). Besides interleukin-17A (IL-17A), Th17 cells also secret IL-17F, IL-21, IL-22 and IL-26, and are marked by CD-161, CCR6, IL-23R and ROR2C $(8,9)$. IL17 induces adjacent cells to produce chemokines, matrix metalloproteinases and anti-microbial peptides, which trigger inflammation and recruit neutrophils and macrophages (10). Previous studies confirmed the infiltration of Th17 cells in the tumors of human and mouse (11). Under the combined stimulus from TGF- $\beta$ and IL-6, the primitive $\mathrm{CD}^{+}{ }^{+} \mathrm{T}$ cells preferentially differentiate into Th17 cells, and IL-23 plays a key role in the maintenance and proliferation of Th17 cells (12).

Although the mechanism remains unknown, the inhibitory effects of Th17 cells on tumor have been reported (13-15). Since the complicated immune-escape mechanism is involved in the tumor inhibition, if Th17 cells serve as a kind of TIL and exert an inhibitory effects on OSCC as they do in other tumors remain elusive. In this study, we detect an aberrant increase and infiltration of Th17 cells in OSCC patients, and then, assess the influence of Th17 cells on OSCC by enhancing their differentiation.

\section{Methods}

The diagram of the experimental procedures could be found in Figure S1.

\section{Tissue specimens}

The blood and tissue specimens were taken from the OSCC patients who got OSCC excision in Chinese PLA General Hospital from May 2016 to May 2017. All OSCC patients were diagnosed as OSCC according to the pathological biopsy. There were 8 OSCC patients donating their blood samples and OSCC tissues. The normal control tissues were collected from the neighbouring tissues at least $0.5 \mathrm{~cm}$ away from the OSCC focus. The control blood specimens were contributed by the health donors in the Infusion Center of Chinese PLA General Hospital. All the patients and healthy donors had no history of radiotherapy, chemotherapy, biotherapy and traditional Chinese medicine treatments before specimen collection (Table 1).

\section{Fluorescence activated cell sorting (FACS)}

FACS was conducted to detect the percentage of the Th17 cells in all $\mathrm{T}$ lymphocytes in the circulating blood and OSCC tissues. The antibodies for cell sorting were mouse anti-human IL-17A Alexa Fluor ${ }^{\circledR} 647$ (Becton, Dickinson and Company, USA) and PerCP mouse antihuman CD4 (Becton, Dickinson and Company, USA). ALEXA 647 mouse IgG1 was used as the isotype control (Becton, Dickinson and Company, USA). The cell sorting was performed and analyzed by flow cytometer (BD FACS Calibur ${ }^{\mathrm{TM}}$ ) as the manufacturer instructed (Becton, Dickinson and Company, USA).

\section{Cell lines}

TCA8113, an OSCC line from human tongue squamous cell carcinoma was preserved by our institute. The vector expressing human IL-17A was purchased from KainuoBio Co., Ltd. (Beijing, China) by inserting the coding sequence of human IL-17A (480bp) into the backbone of pCDNA3.1. The transfection of TCA8113 with the plasmid pcDNA3.1/ IL-17A, and the subsequent screen by G418 were performed as the manufacturer's protocol (Invitrogen, Carlsbad, CA). The surviving cells (TCA8113/pcDNA3.1/ IL-17A) were assessed by ELISA Kit for IL-17A production (U-Cytech, Dutch) and CellTiter $96^{\circledR}$ Aqueous MTS Kit for cell proliferation (Promega, Madison, WI, USA).

\section{Host mice}

The human lymphocytes were extracted from the circulating blood of health donors by Ficoll-Paque density gradient centrifugation as previous described (16). The concentrated lymphocytes were injected at the $3 \times 10^{7}$ cells/mouse into the abdominal cavity of the 4-week-old SCID-beige mouse (average weight is 20-25 g; purchased from Beijing Vital River Laboratory Animal Technology Co., Ltd., Beijing, China) to generate human-PBL-SCID-beige mice. After 4 weeks of injection, the serum was taken from tail vein for human $\operatorname{IgG}$ calibration with the human IgG calibration Kit (MabTech).

\section{OSCC xenograft tumors}

TCA8113/pcDNA3.1-IL-17A, TCA8113/pcDNA3.1 and TCA8113 cells $\left(2.5 \times 10^{6}\right.$ cells/mouse $)$ were, respectively, subcutaneously injected into the left flank of the bodies of the 5-week-old human-PBL-SCID-beige mice to form 
Table 1 General information of patients (P) and donors (D)

\begin{tabular}{lcccccc}
\hline No. & Age (years) & Gender & Smoking & Drink & Cancer & Other disease \\
\hline P1 & 36 & Male & Yes & No & No & No \\
P2 & 61 & Male & Yes & No & No & No \\
P3 & 57 & Female & No & No & No & No \\
P4 & 53 & Male & No & Yes & No & No \\
P5 & 35 & Female & No & No & No & No \\
P6 & 43 & Male & No & No & No & No \\
P7 & 46 & Male & No & No & No & No \\
P8 & 37 & Female & No & No & No & No \\
D1 & 50 & Female & No & No & No & No \\
D2 & 41 & Male & Yes & No & No & No \\
D3 & 30 & Female & No & No & No & No \\
D4 & 34 & Female & No & No & No & No \\
D5 & 38 & Male & No & No & No & No \\
D6 & 39 & Male & Yes & No & No & No \\
D7 & 42 & Male & No & No & No & No \\
D8 & 44 & Male & No & No & No & No \\
\hline
\end{tabular}

xenograft tumors. 27 host mice were randomly assigned into 3 groups. After mice were sacrificed by cervical dislocation, tumors were harvested for following investigations.

\section{Quantitative PCR (Q-PCR)}

Total RNA was extracted from implanted OSCC samples by using trizol total RNA reagent (Invitrogen, Carlsbad, CA). Primers were synthesized by Ao Ke Inc. (Beijing, China), and their sequences are shown as follow: human granzyme B (forward: 5'-GGTGGCTTCCTGATACGA-3' and reverse: 5'-GGATGGGTCTT TTCACAG-3'; human IL-1 $\beta$ (forward: 5'-TACAGTGGCAATGAGGAT-3' and reverse: 5'-AACCAGCATC TTCCTCAG-3'); human IL-6 (forward: 5'-CCACACAGACAGCCACTC-3' and reverse: 5'-TTTGGAAGG TTCAGGTTG-3'); human IL-17A (forward: 5'-TACTACAACCGATCCACCT-3' and reverse: 5'-GGACCA GGATCTCTTGCT-3'); human IL-23A (forward: 5'-ATGATGTTCCCCATATCC-3' and reverse: 5'-TATCA GGGAGCAGAGAGG-3'); human ROR $\gamma$ t (forward: 5'-CTTGGGGAACTGGGACAG-3' and reverse: 5'-GGAGAAGATGTTGGAGCG-3'); human GAPDH (forward: 5'-ATCATCAGCAATGCCTCC-3' and reverse: 5'-AGTCCTTCCACGATACCAA-3'). All the amplifications were conducted in MiniOpticon ${ }^{\mathrm{TM}}$ RealTime PCR system (Bio-Rad Laboratories, USA).

\section{Western blotting}

The procedures for the lysis of the implanted OSCC sample and the manipulation of Western blotting followed those procedures described in previous study (17). The antibodies against human granzyme B (Santa Cruz Biotechnology, Inc. USA), and ROR $\gamma t$ (Merck Millipore, Darmstadt, Germany) were mouse anti-human IgG, while the antibody against human Actin was rabbit IgG (Santa Cruz Biotechnology, Inc. USA). The secondary antibodies conjugated with HRP were goat anti-mouse and goat anti-rabbit IgGs (Santa Cruz Biotechnology, Inc. USA). The ECL chemiluminescent (Thermo Fisher Scientific, USA) was applied for color development. The image analysis was performed with GelPro analyzer4.5 (Media Cybernetics).

\section{ELISA}

The ELISA Kits for human IL-17A (U-CyTech), IL-1 $\beta$ 

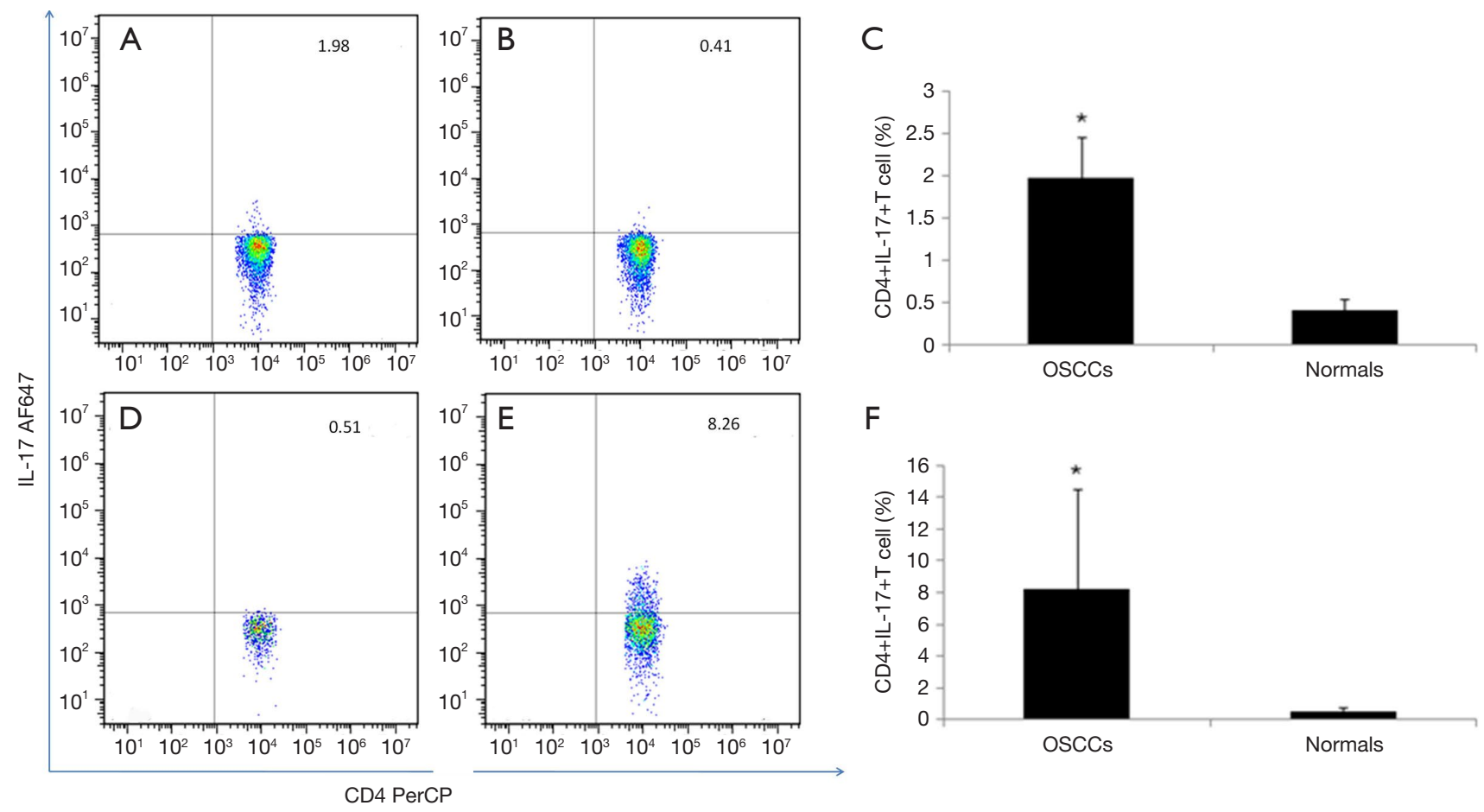

Figure 1 The content of Th17 cells in the OSCC blood and tissues by flow cytometry analysis. (A) Peripheral blood from OSCC patients; (B) peripheral blood from normal donors; (C) statistical analyses for the Th17 percentage in blood samples; (D) normal adjacent tissues from the OSCC focus; (E) tumor tissues from OSCC patients ; $(\mathrm{F})$ statistical analyses for the Th17 percentage in tissue specimens. * $\mathrm{P}<0.05$.

(MabTech), IL-6 (GenWay) and IL-23 (CUSABIO) were purchased from Dakewe Biotech Co., Ltd. (Shenzhen, China). The ELISA manipulation followed the procedures provided by the manufacturers.

\section{Statistical analysis}

The data were analyzed using SPSS 17.0 software (IBM, NY, USA). The SNK test and the Student's $t$-test were used in this study. $\mathrm{P}<0.05$ was regarded as a statistically significant difference.

\section{Results}

The differentiation and infiltration of Th17 cells in OSCC patients were promoted

To address if there was an infiltration of Th17 cells in OSCC samples, FACS assay was employed to enrich Th17 lymphocytes by the antibodies against CD4 and IL17. The percentage of $\mathrm{CD} 4^{+} \mathrm{IL}-17^{+}$cells in the total $\mathrm{CD} 4^{+}$ cells, which represent differentiated Th17 lymphocytes, was $1.98 \% \pm 0.472 \%$ in the peripheral blood of the OSCC patients (Figure 1A), while the counterpart population was only $0.41 \% \pm 0.14 \%$ in the control blood $(\mathrm{P}<0.01$; Figure $1 B, C)$. It suggested that the differentiation of Th17 cells in the OSCC patients was significantly promoted. On the other hand, $8.259 \% \pm 6.25 \%$ cells of the $\mathrm{CD}^{+}$population from OSCC tissues was IL-17 positive (Figure 1D). In contrast, $\mathrm{IL}-17^{+}$cells was only $0.51 \% \pm 0.22 \%$ in all the $\mathrm{CD}^{+}$cells in the normal tissues $(\mathrm{P}<0.05$; Figure $1 E, F)$. This result suggested that the infiltration of Th17 cells took place in the OSCC tissues.

\section{The construction of IL-17A-expressing cell line and tumor-implantation hosts}

The eukaryotic expression vector (pcDNA3.1-IL-17A) was constructed by inserting the full length cDNA of human IL-17A (480 bp) into the backbone of pcDNA3.1 (Figure $2 A, B)$. To get an OSCC line stably expressing IL17A, the pcDNA3.1-IL-17A was transfected into TCA8113 cells (human tongue squamous cell carcinoma). After the screen by G418, the TCA8113 cells stably expressing 

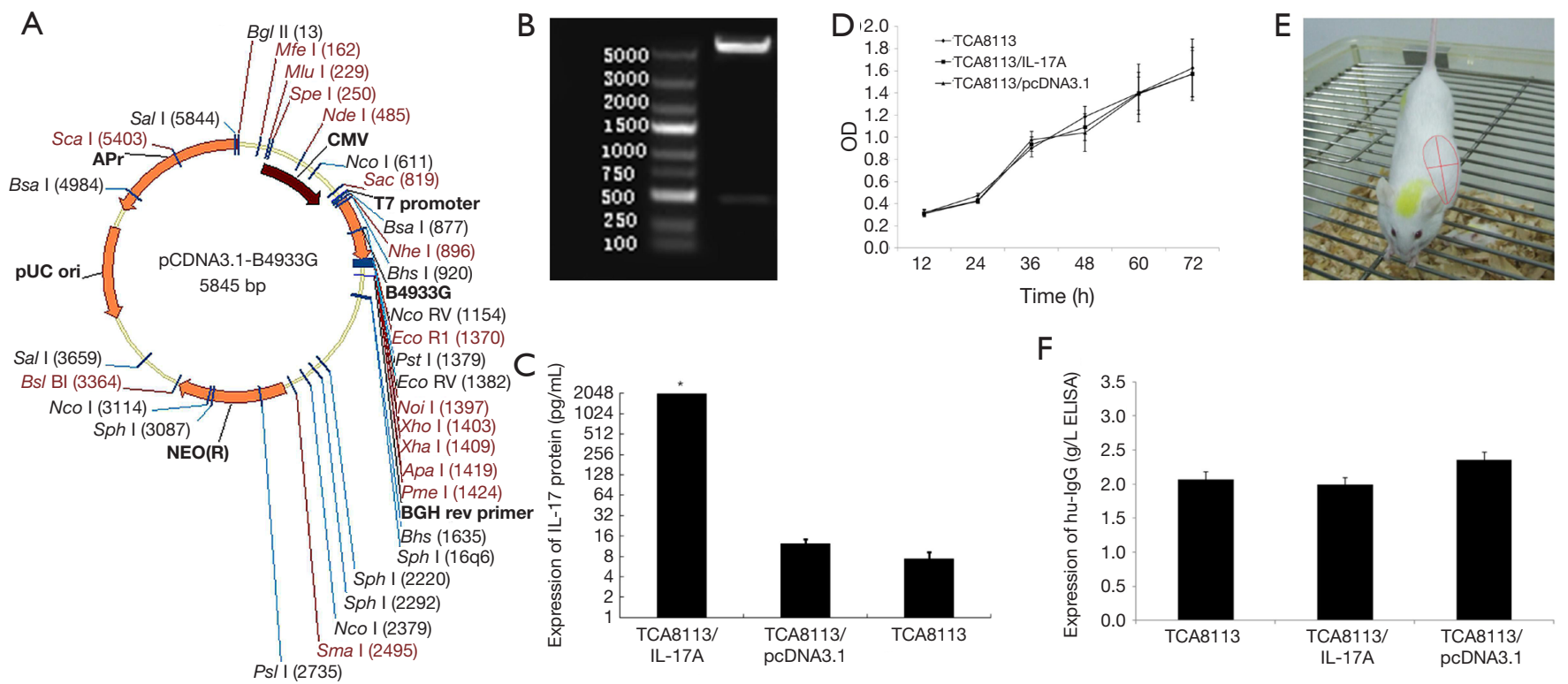

Figure 2 The validity of human-PBL-SCID-beige mice and IL-17A-expressing TCA8113 cell line. (A) The map of pcDNA3.1 vector; (B) identification of pcDNA3.1-IL-17 by NheI/EcoRI cutting; (C) IL-17A production in TCA8113, TCA8113/pcDNA3.1-IL-17A and TCA8113/pcDNA3.1 cells by ELISA assay; (D) cell proliferation of TCA8113, TCA8113/pcDNA3.1-IL-17A and TCA8113/pcDNA3.1 cells by MTS assay; (E) the human- PBL-SCID-beige mouse carrying OSCC xenograft (outlined by red circle); (F) ELISA assay confirmed the human IgG production in human- PBL-SCID-beige mice showed no significant difference. *, $\mathrm{P}<0.05$.

IL-17A was named after TCA8113/pcDNA3.1-IL-17A. ELISA assay confirmed that the average production of IL17A in the TCA8113/pcDNA3.1-IL-17A cell line reached 2,016.256 pg/mL, which was remarkably higher than those on control groups (Figure 2C). Meanwhile, MST test gave rise to the similar re-absorptions among TCA8113/ pcDNA3.1-IL-17A, TCA8113/pcDNA3.1 and TCA8113 groups $(\mathrm{P}>0.05$; Figure $2 \mathrm{D})$, indicating that the secretion of IL-17A exerted little effect on the proliferation of TCA8113/pcDNA3.1-IL-17A cells.

To mimicking the in vivo immune environment of human, human-PBL-SCID-beige mice, in which human immune system was reconstructed, were employed as the hosts for tumor implantation (Figure 2E). All the 27 human-PBLSCID-beige mice were randomly separated into 3 groups. The average concentration of human $\mathrm{IgG}$ in the peripheral blood of the host mice was approximately $2.105 \mathrm{~g} / \mathrm{L}$ and no significant difference among groups (Figure $2 F$ ).

\section{IL-17A inbibited OSCC growth by promoting the differentiation of Th17}

To assess the in vivo effects of IL-17A on OSCC growth, we constructed OSCC implantation model by transplanting the TCA8113/pcDNA3.1-IL-17A cell mass containing $2.5 \times 10^{6}$ cells into human-PBL-SCID-beige mice. During the first 5-9 days after implantation, the tumor size in the TCA8113/pcDNA3.1-IL-17A group differed little from those in TCA8113/pcDNA3.1 and TCA8113 groups (Figure $3 A$ ). From 14 days after implantation on, the tumor sizes in the TCA8113/pcDNA3.1-IL-17A group were noticeably smaller than those in TCA8113/pcDNA3.1 and TCA8113 groups (Figure 3A). In contrast, the tumor sizes in TCA8113/pcDNA3.1 and TCA8113 groups showed few discrepancy from each other in all the period (Figure 3A). After 29 days of implantation, the final volume of the tumor in TCA8113/pcDNA3.1-IL-17A group reached $1.219 \pm 0.626 \mathrm{~cm}^{3}$, which was remarkably smaller than the $2.858 \pm 1.188 \mathrm{~cm}^{3}$ in TCA8113/pcDNA3.1 group or the $2.593 \pm 1.199 \mathrm{~cm}^{3}$ in TCA8113 group (Figure $3 B$ ), indicating that the in vivo growth of OSCC was suppressed by the production of IL-17A.

To verify if IL-17A could enhance the differentiation of Th17, the expression of IL-17A and ROR $\gamma \mathrm{t}$ in the implanted tumors was measured. Q-PCR showed that the transcription of both IL-17A and ROR $\gamma$ t was significantly 

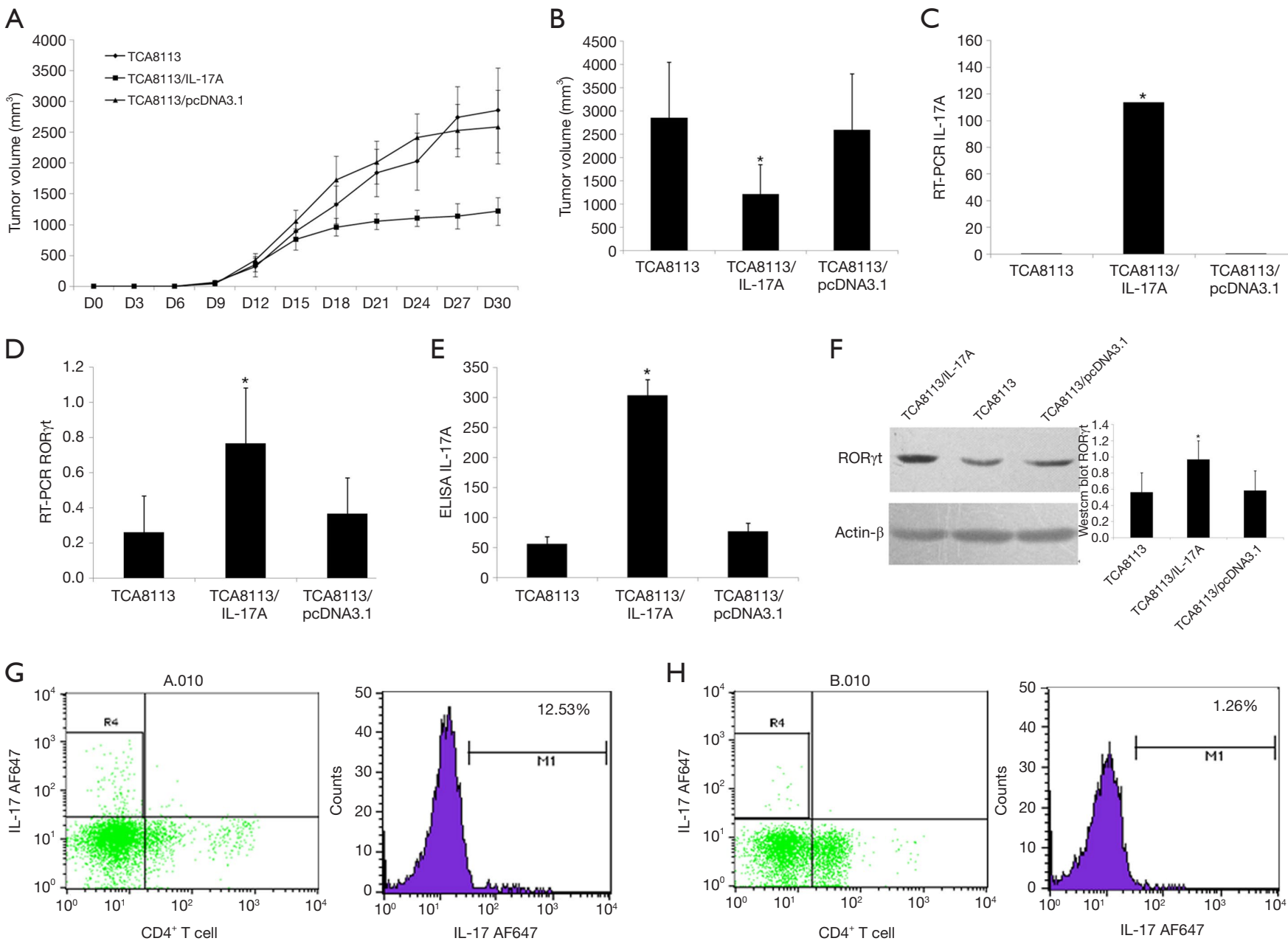

Figure 3 IL-17A impaired OSCC growth and promoted the differentiation of Th17 cells. (A) The growth curves of OSCC xenografts in 30 days after implantation; (B) the average tumor size in TCA8113/pcDNA3.1-IL-17A group was evidently smaller than those in control groups; (C) the IL-17A transcripts in TCA8113/pcDNA3.1-IL-17A group was much higher than those in control groups; (D) the mRNA of ROR $\gamma \mathrm{t}$ in TCA8113/pcDNA3.1-IL-17A group was increased compared with control groups; (E) the protein level of IL-17A in TCA8113/ pcDNA3.1-IL-17A group was much higher than those in control groups; (F) the ROR $\gamma$ t band of Western blotting in TCA8113/pcDNA3.1IL-17A group was significantly denser than those of controls; (G) in a TCA8113/pcDNA3.1-IL-17A samples, the percentage of the Th17 positive cells in all $\mathrm{CD}^{+} \mathrm{T}$ lymphocytes was $12.53 \%$; $(\mathrm{H})$ the percentage of the Th17 positive cells in all CD4 $4^{+} \mathrm{T}$ lymphocytes was only $1.26 \%$ in a TCA8113/pcDNA3.1 sample. *, $\mathrm{P}<0.05$.

increased in the tumors of TCA8113/pcDNA3.1-IL-17A group compared to TCA8113/pcDNA3.1 or TCA8113 group (Figure 3C,D). Consistently, ELISA assay indicated that the IL-17A in TCA8113/pcDNA3.1-IL-17A group was 5-6 folds higher than those in TCA8113/pcDNA3.1 and TCA8113 groups (Figure 3E). Western blotting also exhibited a much stronger band of ROR $\gamma t$ protein in TCA8113/pcDNA3.1-IL-17A group compared to TCA8113/pcDNA3.1 and TCA8113 groups (Figure 3F). To further confirm the enhanced differentiation of Th17 cells in the TCA8113/pcDNA3.1-IL-17A group, the percentage of Th17 cells in all $\mathrm{CD}^{+}{ }^{+} \mathrm{T}$ lymphocytes was measured by FACS. In all the T lymphocytes screened by anti-CD4 antibody, the percentage of Th17 cells was $12.53 \%$ in the TCA8113/pcDNA3.1-IL-17A sample (Figure 3G), while $1.26 \%$ in the TCA8113/pcDNA3.1 sample (Figure $3 H$ ). These findings suggested that IL-17A could suppress OSCC growth by promoting Th17 differentiation. 

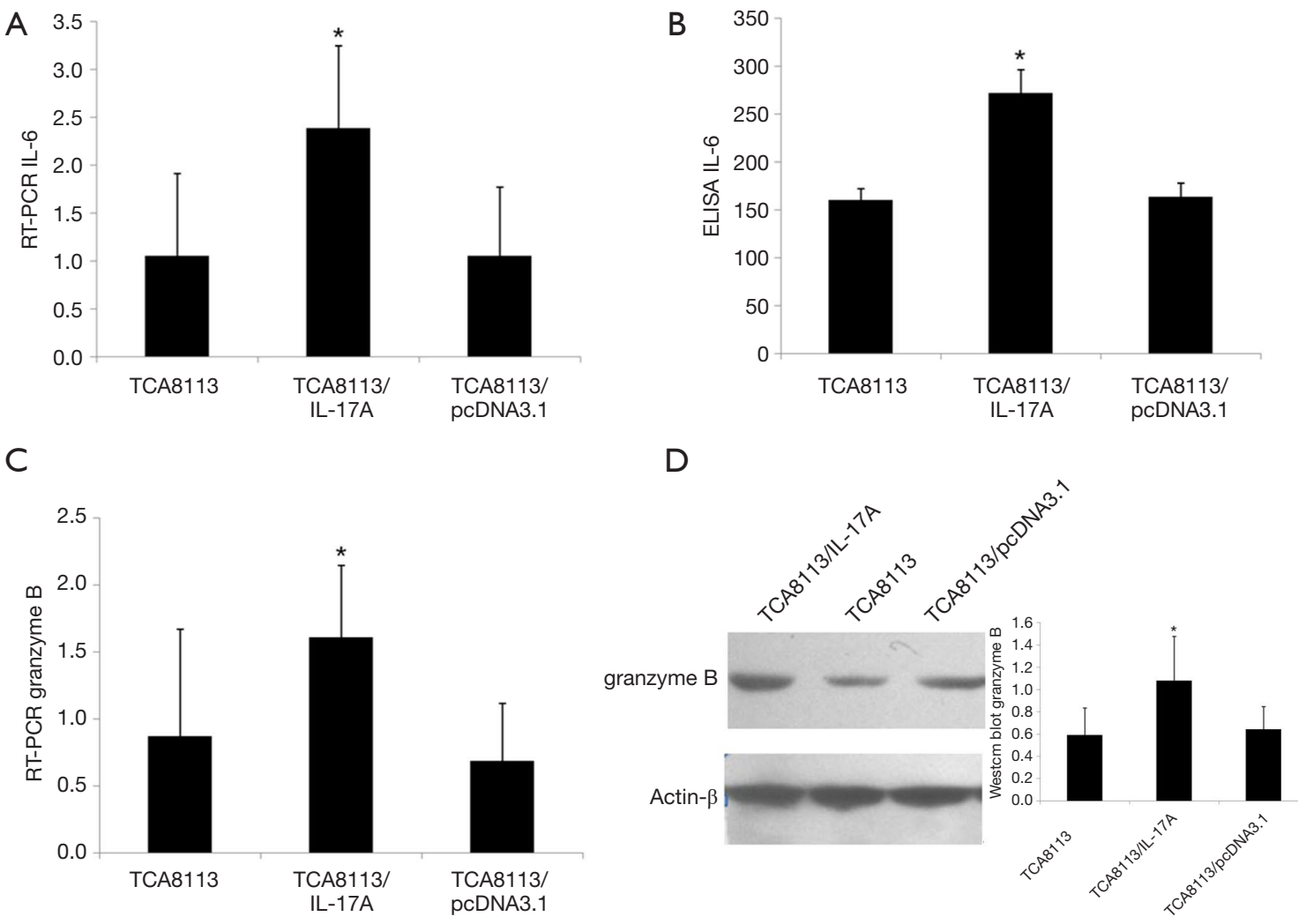

Figure 4 The increased expression of IL-6 and granzyme B in OSCC implants. (A,B) Both the mRNA and the protein level of IL-1 in TCA8113/pCDNA3.1-IL-17A group were significantly higher than those in control groups; (C,D) compared with controls, the transcripts and protein of granzyme B were also elevated in TCA8113/pCDNA3.1-IL-17A group. * $\mathrm{P}<0.05$.

\section{The expression of IL-6 and granzyme B was increased in OSCC implants}

To explore the mechanism how IL-17A induces Th17 differentiation, the expression of IL-1 $\beta$, IL-6 and IL-23A in the OSCC implants was investigated. Q-PCR revealed that only the mRNA of IL-6 was significantly elevated in the TCA8113/pcDNA3.1-IL-17A group (Figure 4A), while the transcription of IL-1 $\beta$ and IL-23A showed no difference (data not shown). ELISA assay confirmed the elevated IL-6 expression in the TCA8113/pcDNA3.1-IL-17A group in contrast to the TCA $8113 / \mathrm{pcDNA} 3.1$ and TCA 8113 groups (Figure 4B). Therefore, it was suggested that the differentiation of Th17 lymphocytes in the OSCC microenvironment was promoted by the elevated IL-6. Moreover, the expression of granzyme B was also examined to explore how Th17 cells repressed OSCC growth. In the TCA8113/ pcDNA3.1-IL-17A group, the transcription of granzyme $\mathrm{B}$ was higher than those in TCA8113/pcDNA3.1 and TCA8113 groups (Figure 4C). Consistently, the Western blotting band of granzyme B in TCA8113/pcDNA3.1IL-17A group was also much denser $(1.081 \pm 0.394)$ than the bands in TCA8113/pcDNA3.1 $(0.646 \pm 0.201)$ and TCA8113 $(0.591 \pm 0.245)$ groups (Figure $4 D)$. These results suggested that differentiated Th17 lymphocytes retarded OSCC growth by recruiting more cytotoxic $\mathrm{T}$ lymphocytes (CTLs) in OSCC micro-environment.

\section{Discussion}

The micro-environment of tumor cells not only decides their survival and growth, but also affects their pharmacological responses to anti-tumor drugs. Tumor micro-environment is constituted by non-tumor cells, extracellular matrix, blood vessels and signaling molecules (18). As one component of the non-tumor cells in tumor micro-environment, the population of the immune cells was a mixture of $\mathrm{T}$ lymphocytes, B lymphocytes, natural killer cells and macrophages. However, the 
infiltrated immune cells were found to play dual roles in tumor progression. The immune responses were putatively regarded to inhibit tumorogenesis, but in the context resembling chronic inflammation, the immune responses promoted malignancy (19). For example, the $\mathrm{CD}^{+}{ }^{+}$Th1 cells function as the major anti-tumor cells by secreting IFN- $\gamma$, while the $\mathrm{CD}^{+}{ }^{+} \mathrm{Th} 2$ cells were reported to prevent or enhance tumor progression in different context (20). Similar to $\mathrm{CD} 4^{+} \mathrm{Th} 2$ cells, the functions of $\mathrm{CD}^{+}{ }^{+} \mathrm{Th} 17$ cells in tumor progression were also controversial based on the different kinds of tumors $(21,22)$.

As the newly discovered subpopulation of $\mathrm{T}$ helper cells, Th17 lymphocytes have been detected in several tumor tissues and circulating blood (6). Previous studies demonstrated that OSCC has a close correlation to immune responses, such as the pre-tumor lesion, lichen planus or vitiligo, in which there is noticeable lymphocyte infiltration (23). Based on above findings, we hypothesized that the immune micro-environment of OSCC was also aberrant as other carcinomas. The increased concentration of Th17 cells detected by FACS in the peripheral blood and tumor tissues from OSCC patients verified the infiltration of Th17 cells in OSCC micro-environment. However, it requires to be elucidated the function of the infiltration of Th17 cells.

The tumors in immune-compromised mice were diminished after inoculated with the ex vivo expanded Th17 cells (24). This finding implicated the anti-tumor activity of Th17 lymphocytes. However, only 10-30\% T lymphocytes differentiated into Th17 cells in $e x$ vivo expansion (25). Hence, such a low ratio limited the application of transfusing the ex vivo expanded Th17 cells into patients because of the scarce sources and expensive price. Fortunately, the proceeding study discovered that Th17 lymphocytes could be induced in the tumor microenvironment and amplified in vivo (25). A variety of cytokines secreted by tumor cells, such as IL-1 $\beta$, IL-23, IL- 6 and TGF- $\beta$, provides a micro-environment facilitating the differentiation of Th17 cells (26). Further investigations found that these cytokines also originated from tumor associated macrophage (TAM), bone marrow-derived suppressing cells (MDSC), antigen present cells (APCs) and fibroblasts (27). IL-17 was reported to stimulate above cells to secrete the cytokines (28). Therefore, we hypothesize that the increase of IL-17 in tumor micro-environment can inhibit tumor growth by promoting Th17 differentiation.

In our study, we generate the human OSCC cell line stably expressing human IL-17A by transfecting TCA8113 with full length cDNA of human IL-17A. By inoculating the IL-17A-expressing TCA8113 cells into humanPBL-SCID-beige mice, we get an artificial environment mimicking human immune response to address if the elevation of IL-17A could enhance the Th17 infiltration in local OSCC micro-environment and to explore the mechanism how Th17 cells suppress OSCC growth. In the TCA8113/pcDNA3.1-IL-17A group, the expression of IL-17A was remarkably higher than those in TCA8113/ pcDNA3.1 and TCA8113 groups. As the marker of Th17 differentiation, the expression of transcription factor ROR $\gamma \mathrm{t}$ was also robustly activated in TCA8113/pcDNA3.1-IL$17 \mathrm{~A}$ group compared to control groups, suggesting that an increased Th17 differentiation resulted from the IL$17 \mathrm{~A}$ stimulus. Finally, the evidently reduced tumor volume in TCA8113/pcDNA3.1-IL-17A group confirmed the inhibitory role of Th17 cells on OSCC growth. On the other hand, we noticed that the growth curve of TCA8113/ pcDNA3.1-IL-17A group was only distinct from those of TCA8113/pcDNA3.1 and TCA8113 groups after 9 days of tumor implantation. This phenomenon may result from the lower production of IL-17A by TCA8113/pcDNA3.1-IL$17 \mathrm{~A}$ cells in the initial stage or the window period required for Th17 differentiation. Since there are few concerns on the differential roles of Th17 cells in the early and later stages of tumors, and/or the dosage-dependent manner of Th17 cells, we plan to clarify those concerns in our further study.

Previous studies disclosed that IL-1 $\beta$, IL-6, IL-21 or IL-23 was necessary for the differentiation of native T cells into Th17 cells (26-28). To clarify the mechanism contributing to the increase of Th17 cells, the expression of above mentioned cytokines was examined in the implanted OSCC samples. The elevated IL-6 expression was detected in the TCA8113/pcDNA3.1-IL-17A group, while the transcription of IL-1 $\beta$ and IL-23A showed no difference among groups. This finding suggested that IL-6 played a critical role in the promoted differentiation of Th17 cells, which was coincided to the previous report (28). Although IL-6 was also reported to enhance the angiogenesis through IL-6/Stat3 pathway in tumors (28), which conflicted to the tumor-suppressing effect in our study, it could be interpreted that the inhibitory effect of Th-17 cells promoted by IL- 6 overwhelmed the angiogenesis stimulated by IL-6. Of course, further study is required to verify this assumption.

Although there were a lot of studies confirmed the inhibition of Th17 lymphocytes on tumor growth $(29,30)$, 
the mechanism still needs to be elucidated. Putatively, Th17 cells were regarded to induce more CTLs against tumor specific-antigen in the local micro-environment (31). So the granzyme B, the hallmark of CTLs, was tested in the implanted OSCC samples. Both Q-PCR and Western blotting results indicated an increase of granzyme $\mathrm{B}$ in the TCA8113/pcDNA3.1-IL-17A cells. Because the repression of the increased activity of CTLs on tumor growth has been established (31), it implicated that the same mechanism was also responsible for the inhibition of Th-17 cells on OSCC growth.

In summary, our study suggested that IL-17 was able to induce the differentiation of Th17 cells by increasing IL- 6 production, and Th17 cells inhibited OSCC growth by exerting the cytotoxic effect. Application of differentiated Th17 cells may greatly retard the growth and reduce the mass and size of OSCC, which would gain more time for patients or decrease the dosage of antitumor drugs. Additionally, the original concentration of the differentiated Th17 cells in the OSCC focus could be exploited as an index for prognosis, in which the more Th17 cells means the better prognosis. Even though, we have to acknowledge that the repression of Th17 cells in OSCC could work through more than the cytotoxic activity, which needs more explorations. Moreover, if Th17 cells have the differential roles in OSCC at different stages also requires further investigation to decide the timing of its clinical administration. Another limitation in the application of Th17 cells is the potential risk of enhancing the progression of tumors other than OSCC, because its function in other tumors has not been clarified yet. Taking above shortcomings into account, the local induction of the differentiation of Th17 cells, instead of the intravenous injection of differentiated Th17 cells would be the preferential approach for OSCC treatment, which has been performed in our study.

\section{Acknowledgments}

Funding: This study was supported by Nursery Funds of Chinese PLA General Hospital (15KMM11).

\section{Footnote}

Conflicts of Interest: All authors have completed the ICMJE uniform disclosure form (available at http://dx.doi. org/10.21037/tcr.2018.06.05). The authors have no conflicts of interest to declare.
Ethical Statement: The authors are accountable for all aspects of the work in ensuring that questions related to the accuracy or integrity of any part of the work are appropriately investigated and resolved. The study was conducted in accordance with the Declaration of Helsinki (as revised in 2013). This study was approved by the Ethical Committee of Chinese PLA (People's Liberation Army) General Hospital. All of the patients participated in this study have signed the informed consent for using their medical records and tissue specimens for research purposes. The animal experiments were approved by the Animal Research Committee at Beijing Laboratory Animal Research Center and carried out in accordance with the authorized Protocol (P2015015).

Open Access Statement: This is an Open Access article distributed in accordance with the Creative Commons Attribution-NonCommercial-NoDerivs 4.0 International License (CC BY-NC-ND 4.0), which permits the noncommercial replication and distribution of the article with the strict proviso that no changes or edits are made and the original work is properly cited (including links to both the formal publication through the relevant DOI and the license). See: https://creativecommons.org/licenses/by-nc-nd/4.0/.

\section{References}

1. Markopoulos AK. Current Aspects on Oral Squamous Cell Carcinoma. Open Dent J 2012; 6:126-30.

2. Mithani SK, Mydlarz WK, Grumbine FL, et al. Molecular genetics of premalignant oral lesions. Oral Dis 2007;13:126-33.

3. Scully C, Bagan J. Oral squamous cell carcinoma overview. Oral Oncol 2009;45:301-8.

4. Jian SL, Hsieh HY, Liao CT, et al. Ga12 Drives Invasion of Oral Squamous Cell Carcinoma through Up-Regulation of Proinflammatory Cytokines. Plos One 2013;8:e66133.

5. Uppaluri R, Dunn GP, Lewis JS Jr. Focus on TILs: prognostic significance of tumor infiltrating lymphocytes in head and neck cancers. Cancer Immun 2008; 8:16.

6. Kesselring R, Thiel A, Pries R, et al. Human Th17 cells can be induced through head and neck cancer and have a functional impact on HNSCC development. Br J Cancer 2010;103:1245-54.

7. Raphael I, Nalawade S, Eagar TN, et al. T cell subsets and their signature cytokines in autoimmune and inflammatory diseases. Cytokine 2015;74:5-17.

8. Bengsch B, Seigel B, Flecken T, et al. Human 
Th17 cells express high levels of enzymatically active dipeptidylpeptidase IV (CD26). J Immunol 2012;188:5438-47.

9. Huh JR, Leung MW, Huang P, et al. Digoxin and its derivatives suppress TH17 cell differentiation by antagonizing ROR $\gamma t$ activity. Nature 2011;472:486-90.

10. Ma Y, Shurin GV, Peiyuan Z, et al. Dendritic cells in the cancer microenvironment. J Cancer 2013;4:36-44.

11. Hoe E, Anderson J, Nathanielsz J, et al. The contrasting role of Th17 immunity in human health and disease. Microbiol Immunol 2017;61:49-56.

12. Ye X, Zhang L, Wang H, et al. The Role of IL-23/ Th17 Pathway in Patients with Primary Immune Thrombocytopenia. PLoS One 2015;10:e0117704.

13. Gnerlich JL, Mitchem JB, Weir JS, et al. Induction of Th17 cells in the tumor micro-environment improves survival in a murine model of pancreatic cancer. J Immunol 2010;185:4063-71.

14. Ye ZJ, Zhou Q, Gu YY, et al. Generation and differentiation of IL-17-producing CD4+ T cells in malignant pleural effusion. J Immunol 2010;185:6348-54.

15. Alizadeh D, Trad M, Hanke NT, et al. Doxorubicin eliminates myeloid-derived suppressor cells and enhances the efficacy of adoptive T-cell transfer in breast cancer. Cancer Res 2014;74:104-18.

16. Rizza P, Santini SM, Logozzi MA, et al. T-cell dysfunctions in hu-PBL-SCID mice infected with human immunodeficiency virus (HIV) shortly after reconstitution: in vivo effects of HIV on highly activated human immune cells. J Virol 1996;70:7958-64.

17. Ma J, Ren Y, Zhang L, et al. Knocking-down of CREPT prohibits the progression of oral squamous cell carcinoma and suppresses cyclin D1 and c-Myc expression. PLoS One 2017;12:e0174309.

18. Wu T, Dai Y. Tumor microenvironment and therapeutic response. Cancer Lett 2017;387:61-8.

19. Hui L, Chen Y. Tumor microenvironment: Sanctuary of the devil. Cancer Lett 2015;368:7-13.

20. Fridman WH, Pagès F, Sautès-Fridman C, et al. The immune contexture in human tumours: impact on clinical outcome. Nat Rev Cancer 2012;12:298-306.

21. von Boehmer H, Daniel C. Therapeutic opportunities for manipulating $\mathrm{T}(\mathrm{Reg})$ cells in autoimmunity and cancer. Nat Rev Drug Discov 2013;12:51-63.

22. Frey DM, Droeser RA, Viehl CT, et al. High frequency of tumor-infiltrating FOXP3(+) regulatory T cells predicts improved survival in mismatch repair-proficient colorectal cancer patients. Int J Cancer 2010;126:2635-43.

23. Douglas WG, Tracy E, Tan D, et al. Development of head and neck squamous cell carcinoma is associated with altered cytokine responsiveness. Mol Cancer Res 2004;2:585-93.

24. Muranski P, Boni A, Antony PA, et al. Tumor-specific Th17-polarized cells eradicate large established melanoma. Blood 2008;112:362-73.

25. Kryczek I, Wei SL, Altuwaijri S, et al. Cutting Edge: Th17 and regulatory $T$ cell dynamics and the regulation by IL-2 in the tumor microenvironment. J Immunol 2007;178:6730-33.

26. Miyahara Y, Odunsi K, Chen W, et al. Generation and regulation of human CD4+ IL-17-producing T cells in ovarian cancer. Proc Natl Acad Sci U S A 2008;105:15505-10.

27. Nagaraj S, Gabrilovich DI. Myeloid-derived suppressor cells in human cancer. Cancer J 2010;16:348-53.

28. Wang L, Yi TM. IL-17 can promote tumor growth through an IL-6-Stat3 signaling pathway. J Exp Med 2009;206:1457-64.

29. Bending D, De la Peña H, Veldhoen M, et al. Highly purified Th17 cells from BDC2.5NOD mice convert into Th1-like cells in NOD/SCID recipient mice. J Clin Invest 2009;119:565-72.

30. Nurieva R, Yang XO, Chung Y, et al. Cutting edge: in vitro generated Th17 cells maintain their cytokine expression program in normal but not lymphopenic hosts. J Immunol 2009;182:2565-8.

31. Martin-Orozco N, Muranski P, Chung Y, et al. T helper 17 cells promote cytotoxic $\mathrm{T}$ cell activation in tumor immunity. Immunity 2009;31:787-98.
Cite this article as: Ren Y, Ma J, Wang T, Bu R, Kong X, Shi Y, Zhang L. Interleukin-17 inhibits the growth of oral squamous cell carcinoma by promoting the differentiation of $\mathrm{T}$ helper 17 cells. Transl Cancer Res 2018;7(4):839-848. doi: 10.21037/ tcr.2018.06.05 
Supplementary
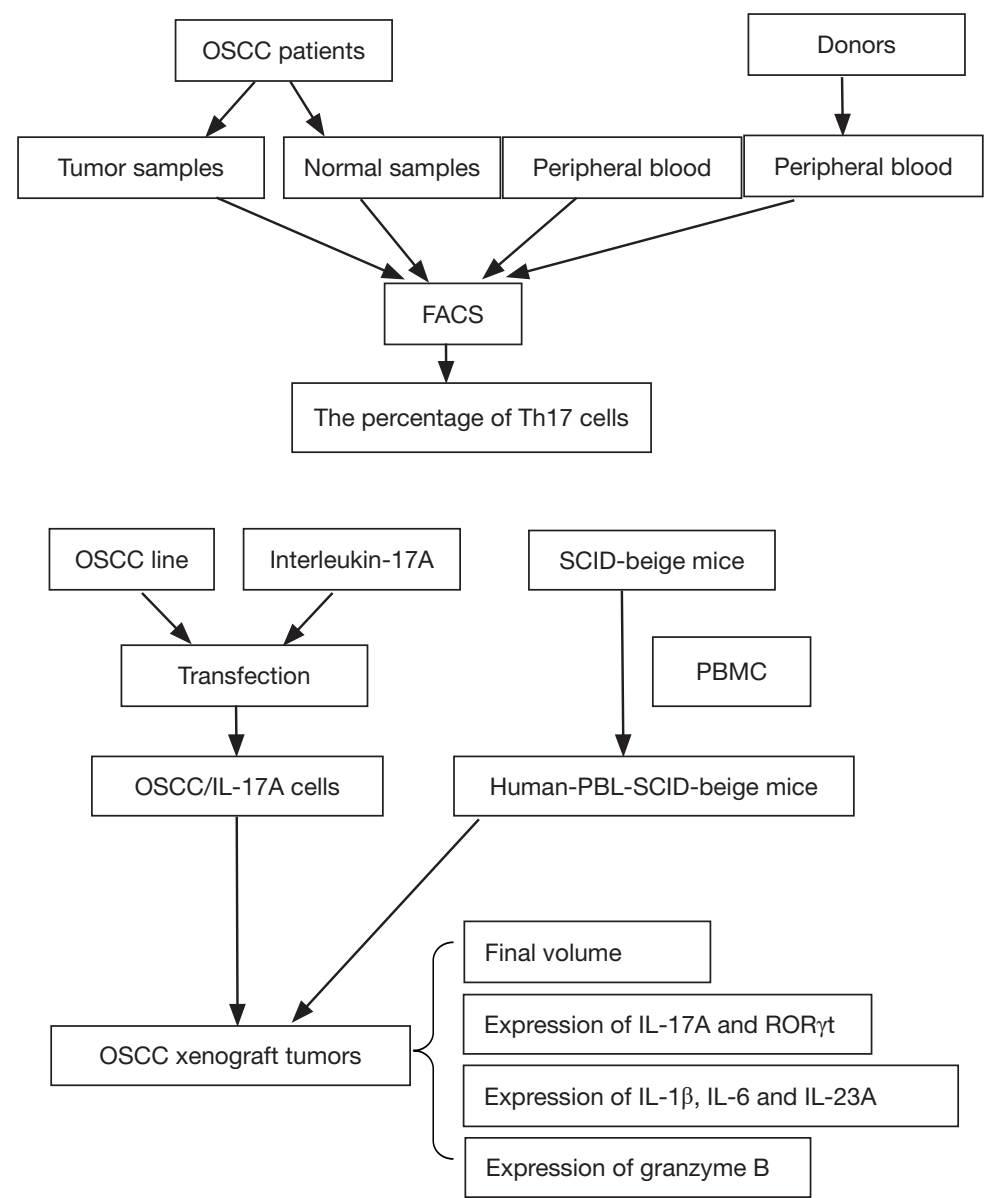

Figure S1 Research process of this study. 